\title{
Effects of Bacillus Thuringiensis Cry Proteins On the Morphology of Western Corn Rootworm (Diabrotica virgifera virgifera) Midgut Cells
}

\author{
Andrew J. Bowling ${ }^{1}$, Heather E. Pence ${ }^{1}$, Alaina M. Turchi ${ }^{2}$, Sek Yee Tan ${ }^{1}$, and Kenneth E. Narva ${ }^{1}$ \\ ${ }^{1 .}$ Dow AgroSciences, Indianapolis, IN, 46268 \\ ${ }^{2 .}$ Indiana University, Bloomington, IN, 47405
}

Western Corn Rootworm (WCR) is a major pest in the United States corn industry, causing annual losses of over one billion dollars [1]. WCR feed on corn roots, which reduces nutrient and water uptake and can cause the plants to lodge, and thus considerably reduces crop yield and quality. One way to combat this pest is the incorporation of genes encoding Bacillus thuringiensis (Bt) crystal (Cry) proteins into the corn genome. Cry proteins interact with the midgut epithelial cells of WCR and form pores in their plasma membranes, disrupting the delicate brush border microvilli, and ultimately causing lysis and/or apoptosis of these cells [2]. Cry-damaged cells induce healing responses in nearby stem cells to repair the midgut in an attempt to prevent death of the insect [3,4]. Herein, we characterize the impact of Cry34/Cry35Ab1 toxins on the anterior midgut morphology of WCR, thereby adding valuable data to our knowledge of the mode-of-action (MOA) for Cry34/Cry35Ab1.

WCR larvae were reared on an artificial diet, and then moved to media overlaid with Cry34/Cry35Ab1 proteins and collected after 24 and 48 hours on the treated diet. Larvae were fixed in $4 \%$ formaldehyde in PBS, dehydrated in a graded ethanol series, infiltrated with LR White, and heat polymerized. Sections were cut $500 \mathrm{~nm}$ thick with a DiATOME Histo diamond knife, stained with toluidine blue O, and imaged with a Leica DM5000 microscope and DFC7000T camera.

Untreated WCR larvae have a classic 'tube within a tube' morphology (Figure 1A). The anterior midgut is composed of a single, smooth layer of columnar cells bearing an apical brush border of microvilli. The medial and posterior midgut cells are characterized by folds and ridges of the apical surface. The lumen of the anterior midgut is dilated and largely clear of material. Between the foregut/esophagus and the anterior midgut is a valve-like structure called the cardiac valve. Food material is relatively sparse in the anterior midgut, but increases in density towards the posterior midgut region. Single peritrophic membrane layers are visible in the anterior midgut, but become multi-lamellar in the medial to posterior midgut regions.

Twenty four hours after feeding on diet treated with Cry34/Cry35Ab1, the epithelial cells of the anterior midgut appear dramatically different (Figure 1B). The apical brush border is no longer contiguous, remaining regions are disrupted and reduced, and cell debris is visible in the lumen. Numerous small, cell-like features surround the outer surface of the midgut; the identity of these structures is currently being investigated. At 48 hours post-feeding, the anterior midgut lumen is nearly completely occluded with cell remnants and swollen epithelial cells (Figure 1C). The cardiac valve has also been negatively impacted by the Cry toxins.

Ingestion of Cry34Ab1 alone appears to cause significant swelling of the midgut epithelial cells, but very little actual bursting of the cells. The microvilli are relatively intact and contiguous, with no noticeable shedding to the lumen (Figure 2A). The lack of extensive cell bursting and/or microvilli shedding leads to a relatively open lumen. The basal region of the epithelial cells is more pronounced, 
possibly as a result of an abnormal extension of the basal labyrinth membranes. The ingestion of Cry35Ab1 alone had very little impact on the morphology of the cells of the midgut, and the alimentary canal as a whole (Figure 2B). However, the microvilli of these cells do appear slightly disrupted, with some secretion of mucous from the foregut cells. The peritrophic membrane is visibly intact and appears normal.

In conclusion, Cry34/Cry35Ab1 proteins offer a robust defense mechanism against WCR. Upon consumption, these proteins disrupt the anterior midgut epithelial cells, resulting in swelling, apoptosis, midgut lumen occlusion, and ultimately death of the insect. These data indicate that Cry protein intoxication may dramatically disrupt the anterior midgut, thereby preventing further ingestion of plant material.

[1] H. Li et al., PLoS ONE 8 (2013)

[2] D. Moellenbeck et al., Nature Biotech 19 (2001), p. 668

[3] A. Castagnola and J. L. Jurat-Fuentes. IOBC/WPRS Bulletin 45 (2009), p. 27

[4] A. Castagnola, S. Eda, and J. L. Jurat-Fuentes. Differentiation 81 (2011), p. 192



Figure 1. Anterior midgut region of 2 nd instar WCR larvae fed $33 \mu \mathrm{g} / \mathrm{cm}^{2}$ Cry34/35Ab1 or untreated diet. A.) Larvae fed untreated diet showing normal midgut epithelial layer, with a clear lumen. B.) Larvae fed $33 \mu \mathrm{g} / \mathrm{cm}^{2}$ Cry34/35Ab1 show extreme blebbing and disorder of the midgut epithelial cells. C.) Larvae fed Cry34/35Ab1 for 48 hours showing highly distorted epithelial cells resulting in a completely occluded lumen. $(\mathrm{CV}=$ cardiac valve; $\mathrm{LU}=$ gut lumen; $\mathrm{CC}=$ columnar cells; $\mathrm{CD}=$ cellular debris; scale bar $=50 \mu \mathrm{m}$ )

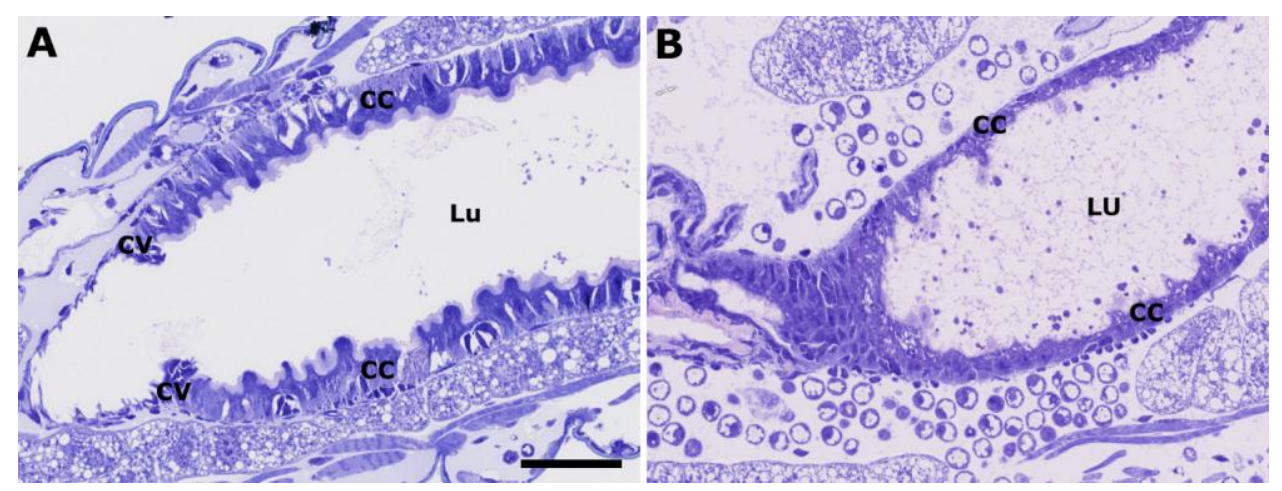

Figure 2. Anterior midgut region of 2nd instar WCR larvae fed either Cry34Ab1 or Cry35Ab1 alone. A.) Larva fed only Cry34Ab1, showing a possible mild effect on the midgut epithelial cells. B.) Larva fed only Cry35Ab1, showing apparently undisturbed midgut epithelial cells. (CV = cardiac valve; $L U=$ gut lumen; $\mathrm{CC}=$ columnar cells; scale bar $=50 \mu \mathrm{m}$ ) 